\title{
Magneto Binary Nanofluid Convection in Porous Medium
}

\author{
Jyoti Sharma, ${ }^{1}$ Urvashi Gupta, ${ }^{2}$ and R. K. Wanchoo ${ }^{2}$ \\ ${ }^{1}$ Energy Research Centre, Panjab University, Chandigarh 160014, India \\ ${ }^{2}$ Dr. S. S. Bhatnagar University Institute of Chemical Engineering and Technology, Panjab University, Chandigarh 160014, India
}

Correspondence should be addressed to Urvashi Gupta; dr_urvashi_gupta@yahoo.com

Received 31 December 2015; Revised 11 March 2016; Accepted 24 March 2016

Academic Editor: Jose C. Merchuk

Copyright (C) 2016 Jyoti Sharma et al. This is an open access article distributed under the Creative Commons Attribution License, which permits unrestricted use, distribution, and reproduction in any medium, provided the original work is properly cited.

\begin{abstract}
The effect of an externally impressed magnetic field on the stability of a binary nanofluid layer in porous medium is considered in this work. The conservation equations related to the system are solved using normal mode technique and Galerkin method to analyze the problem. The complex expressions are approximated to get useful results. Mode of heat transfer is stationary for top heavy distribution of nanoparticles in the fluid layer and top heavy nanofluids are very less stable than regular fluids. Oscillatory motions are possible for bottom heavy distribution of nanoparticles and they are not much influenced by properties of different nanoparticles. A comparative analysis of the instability of water based nanofluids with metallic $(\mathrm{Cu}, \mathrm{Ag})$ and semiconducting $\left(\mathrm{TiO}_{2}\right.$, $\mathrm{SiO}_{2}$ ) nanoparticles under the influence of magnetic field is examined. Semiconducting nanofluids are found to be more stable than metallic nanofluids. Porosity destabilizes the layer while solute difference (at the boundaries of the layer) stabilizes it. Magnetic field stabilizes the fluid layer system significantly.
\end{abstract}

\section{Introduction}

The onset of instability in a horizontal fluid layer, which is heated uniformly from below, has its origin in the experiments of Bénard [1]. He observed that at the onset of instability, the fluid layer resolves into a number of equal cells and they align themselves to form a regular hexagonal pattern. Motivated by the experimental observations of Bénard, the theoretical foundations for a correct interpretation of the problem were laid by Lord Rayleigh [2]. He showed that the convection appears when a certain dimensionless parameter known as Rayleigh number exceeds its critical value. The theory of Rayleigh Bénard convection for a fluid of single component under varying assumptions of hydrodynamics and hydromagnetics is given in the monograph by Chandrasekhar [3]. When the convective motions in the fluid are produced due to two different components, the convection is known as binary convention or double diffusive convection. Stern [4] first established that the interesting effects in binary convection are due to sharp contrast between thermal and solutal diffusivities.

Modern nanotechnology deals with the manufacturing of objects with dimensions of less than hundred nanometers. As a consequence, the idea of suspending these nanoparticles in a base liquid (known as nanofluid) for improving thermal conductivity has been proposed by Choi [5]. He showed a substantial augmentation of heat transport in suspensions of copper or aluminum nanoparticles in water and other liquids. Pioneer works of Masuda et al. [6] and Eastman et al. [7] introduced the thermal conductivity enhancement of nanofluids to the scientific community. Since then, a large number of experimental and theoretical studies have been carried out by numerous researchers. Keblinski et al. [8] and Wang et al. [9] suggested new mechanisms for the transport of thermal energy in nanofluids. An analytical investigation to study the thermal instability of a nanofluid layer using Buongiorno's model [10] was made by Tzou [11] and found that regular fluids exhibit higher stability than nanofluids. Nield and Kuznetsov [12, 13] derived the expression for thermal Rayleigh number for instability of a nanofluid layer in porous medium and conditions for oscillatory motions. Gupta et al. [14] investigated the onset of convection in a horizontal nanofluid layer under vertical magnetic field and found that stability rises with the rise in magnetic field parameter. Rudraiah et al. [15] studied the effect of magnetic field on the convection in a rectangular enclosure and found that the magnetic field reduces the rate of heat transfer. Hayat 
et al. [16] described the heat transfer analysis with thermal radiation on the two-dimensional magnetohydrodynamic flow in a channel with porous walls. Recently, Sheikholeslami et al. [17, 18] investigated MFD viscosity effect and thermal radiation effect on magnetohydrodynamic nanofluid flow numerically using control volume based finite element method, respectively. The effect of rotation on nanofluid convection using Darcy-Brinkman model was considered by Chand and Rana [19]. An expression for thermal Rayleigh number for stationary convection was obtained. A nonlinear study on the nanofluid convection in a rotating porous layer in terms of Nusselt number was made by Bhadauria and Agarwal [20].

For the preparation of nanofluid, when a binary liquid is used, it is termed as binary nanofluid. Instability of a binary nanofluid layer in porous medium was investigated analytically by Kuznetsov and Nield [21]. The results were approximated by limiting to the case of large nanoparticle Lewis number and large Prandtl number. Further, Gupta et al. [22] and Yadav et al. [23] studied the same problem analytically as well as numerically for alumina-water nanofluid independent of the restrictions on parameters. The influence of magnetic field on binary nanofluid convection was considered by Gupta et al. [24]. They have made valid approximations in the complex expressions for analytical study.

Convection in binary nanofluids with magnetic field has its importance in enhanced oil recovery from underground reservoirs which motivated us to study the influence of magnetic field on the stability of a binary nanofluid layer in porous medium. Darcy revealed proportionality between flow rate and applied pressure difference for a fluid flowing through porous medium which is used in the model under investigation. The nanoscale effects, Brownian motion (movement of nanoparticles from high concentration to low concentration) and thermophoresis (movement of nanoparticles from high temperature site to low temperature site), play an important role in enhancement of effective thermal conductivity of the fluid. Thus the partial differential equations incorporating both the effects of Brownian motion and thermophoresis are considered. Also, the presence of solute (with much lower diffusivity than that of nanoparticles and heat) in nanofluid layer makes the convection problem a triple diffusion process resulting in the introduction of three Rayleigh numbers due to heat, solute, and nanoparticles. The problem is solved using normal mode analysis and residuals method to get an eigenvalue equation which is further analyzed for stationary and oscillatory mode of convection in the fluid layer. Complex expressions are simplified using approximations on the parameters involved. Mathematica software is used to plot the stability curves for the impact of magnetic field, porosity, solute concentration difference, nanoparticle volume fraction difference, Dufour parameter, and Soret parameter on metallic $(\mathrm{Cu}, \mathrm{Ag})$ and semiconducting $\left(\mathrm{TiO}_{2}\right.$, $\mathrm{SiO}_{2}$ ) nanoparticles.

\section{Problem Formulation}

Let us consider a horizontal binary nanofluid layer of thickness $d$ in the presence of uniform magnetic field $\left(0,0, h_{0}\right)$.
The temperature, solute concentration, and nanoparticle volume fraction at the lower and upper layers are taken to be $T_{1}$ and $T_{0}, C_{1}$ and $C_{0}$, and $\phi_{0}$ and $\phi_{1}$, respectively, with $T_{1}>T_{0}, C_{1}>C_{0}$, and $\phi_{1}>\phi_{0}$. The basic equations which express the interaction between the fluid motion and the magnetic field as given by Buongiorno [10], Kuznetsov and Nield [21], and Gupta et al. [24] are

$$
\begin{aligned}
& \nabla \cdot \mathbf{u}_{D}=0 \\
& \frac{\partial \phi}{\partial t}+\frac{\mathbf{u}_{D}}{\varepsilon} \cdot \nabla \phi=\nabla \cdot\left[D_{B} \nabla \phi+D_{T} \frac{\nabla T}{T}\right], \\
& 0=-\nabla p-\frac{\mu}{k} \mathbf{u}_{D}+\left[\phi \rho_{p}\right. \\
& \left.+(1-\phi)\left\{\rho\left(1-\beta_{T}\left(T-T_{0}\right)-\beta_{C}\left(C-C_{0}\right)\right)\right\}\right] \mathbf{g} \\
& +\frac{\mu_{e}}{4 \pi}(\mathbf{h} \cdot \nabla) \mathbf{h} \\
& (\rho c)_{m} \frac{\partial T}{\partial t}+(\rho c)_{f} \mathbf{u}_{D} \cdot \nabla T=\left(k_{m} \nabla^{2} T\right)+\varepsilon(\rho c)_{p} \\
& \cdot\left[D_{B} \nabla \phi \cdot \nabla T+D_{T} \frac{\nabla T \cdot \nabla T}{T}\right]+\rho c D_{T C} \nabla^{2} C, \\
& \frac{\partial C}{\partial t}+\frac{\mathbf{u}_{D}}{\varepsilon} \cdot \nabla C=D_{S} \nabla^{2} C+D_{C T} \nabla^{2} T,
\end{aligned}
$$

where $\mathbf{u}_{D}, C, T, \phi, D_{B}, D_{T}, D_{S}, D_{T C}, D_{C T}, \mu, \mathbf{h}, \mu_{e},(\rho c)_{f}$, $(\rho c)_{p},(\rho c)_{m}, k_{m}, \varepsilon, k, \rho_{f}, t, \mathbf{g}, \beta_{T}$, and $\beta_{C}$ denote, respectively, the Darcy velocity, concentration of solute, temperature, volume fraction of nanoparticles, Brownian diffusion coefficient, thermophoretic diffusion coefficient, solute diffusivity coefficient, Dufour diffusivity, Soret diffusivity, fluid viscosity, magnetic field, magnetic permeability, fluid heat capacity, nanoparticles heat capacity, medium heat capacity, thermal conductivity of the medium, porosity, permeability of the medium, base-fluid's density, time, acceleration due to gravity, thermal volumetric coefficient, and solutal volumetric coefficient. The Maxwell's equations are

$$
\begin{aligned}
\frac{\partial \mathbf{h}}{\partial t}+\left(\frac{\mathbf{u}_{D}}{\varepsilon} \cdot \nabla\right) \mathbf{h} & =(\mathbf{h} \cdot \nabla) \frac{\mathbf{u}_{D}}{\varepsilon}+\eta \nabla^{2} \mathbf{h}, \\
\nabla \cdot \mathbf{h} & =0,
\end{aligned}
$$

where $\eta$ is the resistivity of the fluid. Let us consider the nondimensional variables as

$$
\begin{aligned}
\left(x^{*}, y^{*}, z^{*}\right) & =\frac{(x, y, z)}{d}, \\
t^{*} & =\frac{t \alpha_{m}}{\sigma d^{2}}, \\
\mathbf{u}^{*} & =\mathbf{u}_{D} \frac{d}{\alpha_{m}}, \\
p^{*} & =\frac{p k}{\mu \alpha_{m}}, \\
\mathbf{h}^{*} & =\frac{\mathbf{h}}{h_{0}},
\end{aligned}
$$




$$
\begin{aligned}
\phi^{*} & =\frac{\phi-\phi_{1}}{\phi_{1}-\phi_{0}}, \\
C^{*} & =\frac{C-C_{0}}{C_{1}-C_{0}}, \\
T^{*} & =\frac{T-T_{0}}{T_{1}-T_{0}},
\end{aligned}
$$$$
\text { with } \alpha_{m}=\frac{k_{m}}{(\rho c)_{f}}, \sigma=\frac{(\rho c)_{m}}{(\rho c)_{f}} \text {. }
$$

Using nondimensional variables, (1)-(7) (after removing the asterisks) now become

$$
\begin{aligned}
& \nabla \cdot \mathbf{u}= 0, \\
& 0=-\nabla p-\mathbf{u}-\operatorname{Rm} \widehat{k}-\operatorname{Rn} \phi \widehat{k}+R_{a} T \widehat{k} \\
&+\frac{\mathrm{Rs}}{\mathrm{Le}} C \widehat{k}+Q \frac{\operatorname{Pr}_{1}}{\operatorname{Pr}_{2}}(\mathbf{h} \cdot \nabla) \mathbf{h}, \\
& \frac{\partial T}{\partial t}+\mathbf{u} \cdot \nabla T=\nabla^{2} T+\frac{N_{B}}{\mathrm{Ln}} \nabla \phi \cdot \nabla T+\frac{N_{A} N_{B}}{\mathrm{Ln}} \nabla T \\
& \frac{1}{\sigma} \frac{\partial \phi}{\partial t}+\frac{1}{\varepsilon} \mathbf{u} \cdot \nabla \phi= \frac{1}{\mathrm{Ln}} \nabla^{2} \phi+\frac{N_{A}}{\mathrm{Ln}^{2}} \nabla^{2} T, \\
& \frac{1}{\sigma} \frac{\partial C}{\partial t}+\frac{1}{\varepsilon} \mathbf{u} \cdot \nabla C= \frac{1}{\mathrm{Le}} \nabla^{2} C+S_{C T} \nabla^{2} T, \\
& \frac{\partial \mathbf{h}}{\partial t}+\frac{1}{\varepsilon}(\mathbf{u} \cdot \nabla) \mathbf{h}=(\mathbf{h} \cdot \nabla) \frac{1}{\varepsilon} \mathbf{v}+\frac{\operatorname{Pr}_{1}}{\operatorname{Pr}_{2}} \nabla^{2} \mathbf{h},
\end{aligned}
$$

where $\operatorname{Pr}_{1}=\mu / \rho_{f} \alpha_{m}, \operatorname{Ln}=\alpha_{m} / D_{B}, R_{a}=\rho \mathbf{g} \beta_{T} \kappa d\left(T_{1}-\right.$ $\left.T_{0}\right) / \mu \alpha_{m}, \mathrm{Rm}=\left[\rho_{p} \phi_{0}+\rho\left(1-\phi_{0}\right)\right] \mathbf{g} \kappa d / \mu \alpha_{m}, \mathrm{Le}=\alpha_{m} / D_{S}, \mathrm{Rs}=$ $\rho \mathbf{g} \beta_{C} \kappa d\left(C_{1}-C_{0}\right) / \mu D_{S}, \operatorname{Rn}=\left(\rho_{p}-\rho\right)\left(\phi_{1}-\phi_{0}\right) \mathbf{g} \kappa d / \mu \alpha_{m}, N_{A}=$ $D_{T}\left(T_{1}-T_{0}\right) / D_{B} T_{0}\left(\phi_{1}-\phi_{0}\right), N_{B}=\left(\varepsilon(\rho c)_{P} / \rho c\right)\left(\phi_{1}-\phi_{0}\right), S_{T C}=$ $D_{T C}\left(C_{1}-C_{0}\right) / \alpha_{m}\left(T_{1}-T_{0}\right)$, and $S_{C T}=D_{C T}\left(T_{1}-T_{0}\right) / \alpha_{m}\left(C_{1}-\right.$ $C_{0}$ ) are the Prandtl number, the nanofluid Lewis number, the thermal Rayleigh number, the basic density Rayleigh number, the solute Lewis number, the solute Rayleigh number, the concentration Rayleigh number, the diffusivity ratio, the particle-density increment, regular Dufour parameter, and regular Soret parameter, respectively. Due to the inclusion of magnetic field, we have two additional parameters: the magnetic Prandtl number and the Chandrasekhar number defined as $\operatorname{Pr}_{2}=\mu / \rho \eta$ and $Q=\mu_{e} h_{o}{ }^{2} k / 4 \pi \eta \mu$, respectively.

\section{Normal Mode Technique and Galerkin Method}

Initially, the fluid layer is assumed to be in a state of rest. Therefore the basic solution as derived from (9) becomes

$$
\begin{aligned}
T_{i} & =1-z, \\
C_{i} & =1-z, \\
\phi_{i} & =z,
\end{aligned}
$$

where the subscript " $i$ ” represents the initial variable. Let us introduce small perturbations on the initial state as

$$
\begin{aligned}
& \mathbf{u}=0+\widetilde{\mathbf{u}}, \\
& p=p_{i}+\widetilde{p}, \\
& T=T_{i}+\widetilde{T}, \\
& C=C_{i}+\widetilde{C}, \\
& \phi=\phi_{i}+\widetilde{\phi}, \\
& \mathbf{h}=(0,0,1)+\widetilde{\mathbf{h}},
\end{aligned}
$$

where the superscript “ " denotes the perturbed variable. Using (11) on the set of (9), we get a new set of equations known as perturbation equations. To solve perturbation equations, normal mode analysis is used as follows:

$$
(\widetilde{w}, \widetilde{T}, \widetilde{C}, \widetilde{\phi})=(W, T, \Psi, \Phi)(z) e^{i l x+i k y+n t},
$$

where $l x$ and $k y$ are the wave numbers along $x$ and $y$ directions, respectively, and $n$ is the growth rate. For neutral stability, let us write $n=i \omega$; $\omega$ is a real number and represents the frequency of oscillation. The conditions for two free boundaries are

$$
\begin{aligned}
W & =0, \\
D^{2} W & =0, \\
T & =0, \\
\Psi & =0, \\
\Phi & =0
\end{aligned}
$$

$$
\text { at } z=0, z=1 \text {, with } D \equiv \frac{d}{d z} \text {. }
$$

The Galerkin weighted residual method is employed to determine the eigenvalue equation which gives

$$
(W, T, \Psi, \Phi)=\left(A_{1}, B_{1}, C_{1}, D_{1}\right) \sin \pi z,
$$

satisfying the boundary conditions given by (13). Using (14) in perturbation equations produces the eigenvalue equation for the system as

$$
\begin{aligned}
& \operatorname{Rn} \alpha^{2}\left(\varepsilon J+\frac{\varepsilon}{\sigma} \frac{\operatorname{Pr}_{2}}{\operatorname{Pr}_{1}} n\right)\left(\frac{J^{2}+J n \mathrm{Le}+J n-J^{2} S_{C T} S_{T C} \mathrm{Le}}{\varepsilon}\right. \\
&+\left.\frac{n^{2} \mathrm{Le}}{\sigma}+\frac{N_{A}}{\mathrm{Ln}} J\left(J+\frac{n \mathrm{Le}}{\sigma}-\frac{J S_{T C} \mathrm{Le}}{\varepsilon}\right)\right)-\left(\frac{n}{\sigma}\right. \\
&+\left.\frac{J}{\mathrm{Ln}}\right)\left(J\left(\varepsilon J+\frac{\varepsilon}{\sigma} \frac{\operatorname{Pr}_{2}}{\operatorname{Pr}_{1}} n\right)+Q \pi^{2} J\right)\left(J^{2}+\frac{J n}{\sigma}+J n\right. \\
&+\left.\frac{n^{2}}{\sigma}-J^{2} S_{C T} S_{T C} \operatorname{Le}\right)+\operatorname{Rs} \alpha^{2}\left(\frac{n}{\sigma}+\frac{J}{\mathrm{Ln}}\right)(\varepsilon J \\
&+\left.\frac{\varepsilon}{\sigma} \frac{\operatorname{Pr}_{2}}{\operatorname{Pr}_{1}} n\right)\left(\frac{J+n}{\varepsilon}-J S_{C T}\right)+R_{a} \alpha^{2}\left(\varepsilon J+\frac{\varepsilon}{\sigma} \frac{\operatorname{Pr}_{2}}{\operatorname{Pr}_{1}} n\right) \\
& \cdot\left(J+\frac{n}{\sigma}-\frac{J S_{T C} \mathrm{Le}}{\varepsilon}\right)\left(\frac{n}{\sigma}+\frac{J}{\mathrm{Ln}}\right)=0, \\
& \text { where } \alpha=\left(l x^{2}+k y^{2}\right)^{1 / 2}, J=\pi^{2}+\alpha^{2} .
\end{aligned}
$$




\section{Results and Discussion}

4.1. Nonoscillatory Convection. For stationary convection, put $\omega=0$ in (15). This gives the Rayleigh number as

$$
\begin{aligned}
R_{a} & =\frac{1}{\left(\varepsilon-\operatorname{Le} S_{T C}\right)}\left[\left(\frac{\left(\pi^{2}+\alpha^{2}\right)^{2} \varepsilon}{\alpha^{2}}+\frac{\left(\pi^{2}+\alpha^{2}\right) Q \pi^{2}}{\alpha^{2}}\right.\right. \\
& \left.-\operatorname{RnLn})\left(1-S_{C T} S_{T C} \mathrm{Le}\right)-\operatorname{Rs}\left(1-\varepsilon S_{C T}\right)\right] \\
& -\operatorname{Rn} N_{A} .
\end{aligned}
$$

Note that (16) attains its minimum when $x^{2}=Q / \varepsilon+1$ with $x=\alpha^{2} / \pi^{2}$. Thus the critical wave number increases with increase in Chandrasekhar number and decreases with increase in porosity while it is independent of the presence of solute and nanoparticles. When the base fluid is one component in nonporous medium $\left(S_{T C}, S_{C T}\right.$, and Rs are zeros; $\varepsilon$ is unity), (16) reduces to

$$
\begin{aligned}
R_{a}= & \frac{\left(\pi^{2}+\alpha^{2}\right)}{\alpha^{2}}\left[\left(\pi^{2}+\alpha^{2}\right)^{2}+Q \pi^{2}\right] \\
& -\operatorname{Rn}\left(\operatorname{Ln}+N_{A}\right),
\end{aligned}
$$

which agrees with the results given by Gupta et al. [14] for the instability in nanofluids with magnetic field.

The presence of magnetic field delays the onset of convection as coefficient of $Q$ in (16) is positive. Also, for the large value of $\mathrm{Rn}$, Rayleigh number $R_{a}$ takes negative value meaning thereby that the top heavy arrangement of nanoparticles makes the system very unstable. Also $N_{B}$ does not appear in the expression for $R_{a}$ and $N_{A}$ appears only with $\mathrm{Rn}$. This is due to the fact that the regular cross-diffusion terms dominate the nanofluid cross-diffusion terms. It is worthwhile to mention that effect of $N_{A}$ on $R_{a}$ is much less than $\mathrm{Rn}$ meaning thereby that the influence of density of nanoparticles on the stability is more than their conductivity.

4.2. Oscillatory Convection. For oscillatory convection $\omega \neq 0$. Let us substitute $n=i \omega$ in (15) to find the Rayleigh number for oscillatory motions. As the binary nanofluid convection is a triple diffusion process, the expression for thermal Rayleigh number for oscillatory convection is complex. Thus in order to analyze the problem analytically, let us make valid approximations as Lewis number and Prandtl number approach to infinity, Dufour and Soret parameters are negligible, and heat capacity ratio is unity. Thus the real and imaginary parts of (15) reduce to

$$
\begin{aligned}
& \frac{2}{\alpha^{2}}\left(J^{2}+\frac{J \pi^{2} \mathrm{Q}}{\varepsilon}\right)=R_{a}-\frac{J^{2} \mathrm{Rn}}{\varepsilon \omega^{2}}+\frac{\mathrm{Rn}}{\varepsilon}+\frac{\mathrm{Rs}}{\varepsilon}, \\
& \frac{1}{\alpha^{2}}\left(1-\frac{\omega^{2}}{J^{2}}\right)\left(J^{2}+\frac{J \pi^{2} \mathrm{Q}}{\varepsilon}\right)=R_{a}+\frac{2 \mathrm{Rn}}{\varepsilon}+\frac{\mathrm{Rs}}{\varepsilon} .
\end{aligned}
$$

Equations (18) and (19) give

$$
\omega^{2}=-a^{2} \operatorname{Rn}\left(\varepsilon\left(1+\frac{\pi^{2} Q}{\varepsilon J}\right)\right)^{-1} .
$$

Note that $\omega^{2}$ is negative when $\mathrm{Rn}$ is positive meaning thereby that the oscillatory mode of heat transfer is not possible for top heavy nanoparticle distribution in the fluid layer. This was expected as for oscillations to occur two of the buoyancy forces must act in opposite directions. Also, the value of $\omega^{2}$ diminishes with the rise in Chandrasekhar number and porosity while it is independent of presence of solute in the fluid layer. Using (20) in (18) gives

$$
\begin{aligned}
R_{a} \text { (Oscillatory) }= & \frac{\left(\pi^{2}+\alpha^{2}\right)^{2}}{\alpha^{2}}+\frac{\left(\pi^{2}+\alpha^{2}\right) Q \pi^{2}}{\alpha^{2} \varepsilon} \\
& -\frac{\mathrm{Rn}}{\varepsilon}-\frac{\mathrm{Rs}}{\varepsilon} .
\end{aligned}
$$

The expression for $R_{a}$ on neglecting Dufour and Soret parameters in (16) becomes

$$
\begin{aligned}
R_{a} \text { (Stationary) } & \\
= & {\left[\left(\frac{\left(\pi^{2}+\alpha^{2}\right)^{2}}{\alpha^{2}}+\frac{\left(\pi^{2}+\alpha^{2}\right) Q \pi^{2}}{\alpha^{2} \varepsilon}-\frac{\mathrm{Rn} \operatorname{Ln}}{\varepsilon}\right)\right.} \\
& \left.-\frac{\mathrm{Rs}}{\varepsilon}\right]-\operatorname{Rn} N_{A} .
\end{aligned}
$$

For $\mathrm{Rn}$ negative, the value of $R_{a}$ for oscillatory convection is less than that for stationary convection. Thus mode of convection must be oscillatory for bottom heavy distribution of nanoparticles in the fluid layer as variations in the density caused by heat compete with the density gradient caused by nanoparticles and solute concentration at the bottom of the layer which makes bottom heavy nanofluids more stable than regular fluids. Also, coefficient of $\mathrm{Rn}$ is very small in (21) which establishes the result that oscillatory mode of convections is not much influenced by the presence of nanoparticles at the bottom of the layer.

It is interesting to note that while both the nanoscale effects (thermophoresis and Brownian motion) are responsible for the convection in a nanofluid layer, thermophoresis at a higher value of thermophoretic diffusivity is found to have more influence on the value of Rayleigh number and hence on the stability of the nanofluids. Also, note that the radius of nanoparticles appears in the expression of Brownian diffusion coefficient (refer Gupta et al. [14]) and the effects of Lewis number and diffusivity ratio are stabilizing/destabilizing for bottom/top heavy configuration of nanoparticles and hence increase in the radius of nanoparticles makes the system more stable/unstable for bottom/top heavy nanofluids.

\section{Numerical Results and Discussion}

Keeping in mind the fact that oscillatory motions are not much influenced by the properties of different nanoparticles, 


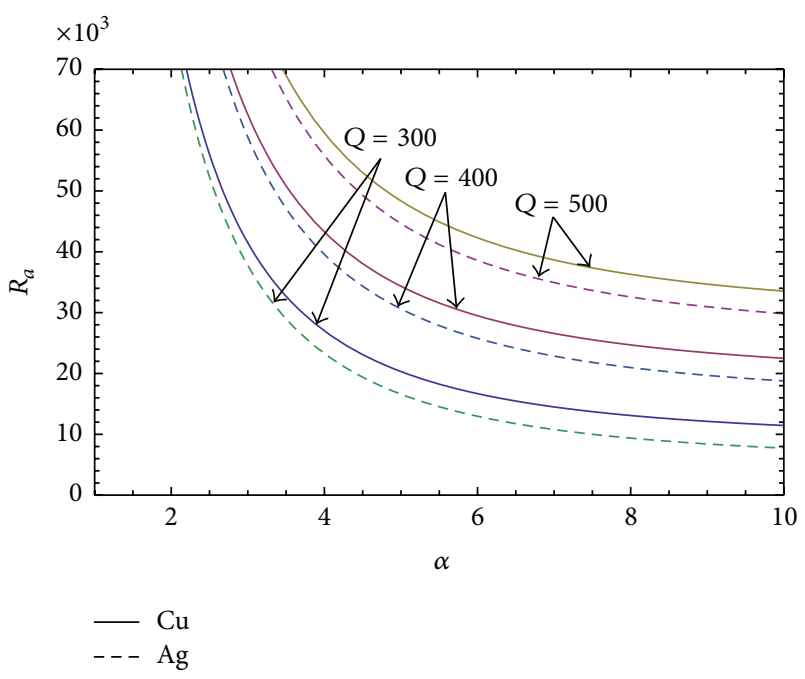

Figure 1: Effect of $Q$ on metallic nanoparticles.

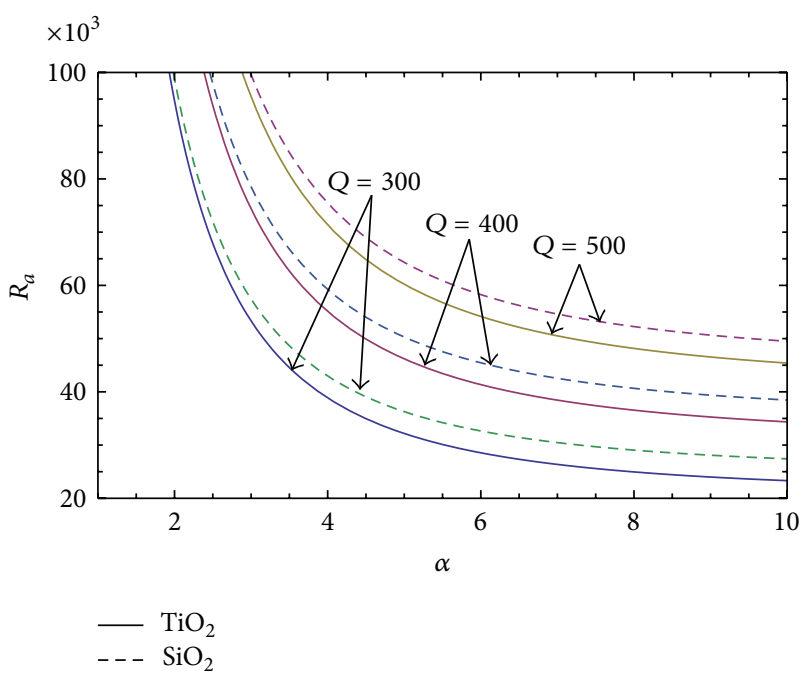

FIGURE 2: Effect of $Q$ on semiconducting nanoparticles.

the stability curves are drawn for stationary mode of heat transfer (see (16)) with the help of Mathematica software. Figures 1-12 show the role played by magnetic field, porosity, solute concentration difference, nanoparticle volume fraction difference, Dufour parameter, and Soret parameter on Rayleigh number for metallic ( $\mathrm{Cu}, \mathrm{Ag}$ ) and nonmetallic $\left(\mathrm{TiO}_{2}, \mathrm{SiO}_{2}\right)$ nanoparticles in water based nanofluids. Table 1 shows the physical properties of metallic and nonmetallic nanoparticles considered in the study.

In (16), we have three parameters $\mathrm{Ln}, \mathrm{Rn}$, and $N_{A}$ which depend on nanofluid properties. According to Buongiorno [10], for water based nanofluid, $\operatorname{Ln}=5000$. Using Table 1, values of nanofluid parameters $\mathrm{Rn}$ and $N_{A}$ are found as shown in Table 2. Note that the higher values of nanoparticle Rayleigh number lead to negative values of thermal Rayleigh number and hence system becomes unstable. Thus the values of nanoparticle Rayleigh number are taken to be small as these values can be controlled with the depth of the layer and
TABLE 1: Physical properties of water and nanoparticles under consideration [25].

\begin{tabular}{lccccc}
\hline Physical properties & Water & $\mathrm{Cu}$ & $\mathrm{Ag}$ & $\mathrm{TiO}_{2}$ & $\mathrm{SiO}_{2}$ \\
\hline$\rho\left(\mathrm{Kg} / \mathrm{m}^{3}\right)$ & 997.1 & 8933 & 10500 & 4250 & 2600 \\
$k(\mathrm{~W} / \mathrm{mK})$ & 0.613 & 401 & 429 & 8.9 & 10.4 \\
\hline
\end{tabular}

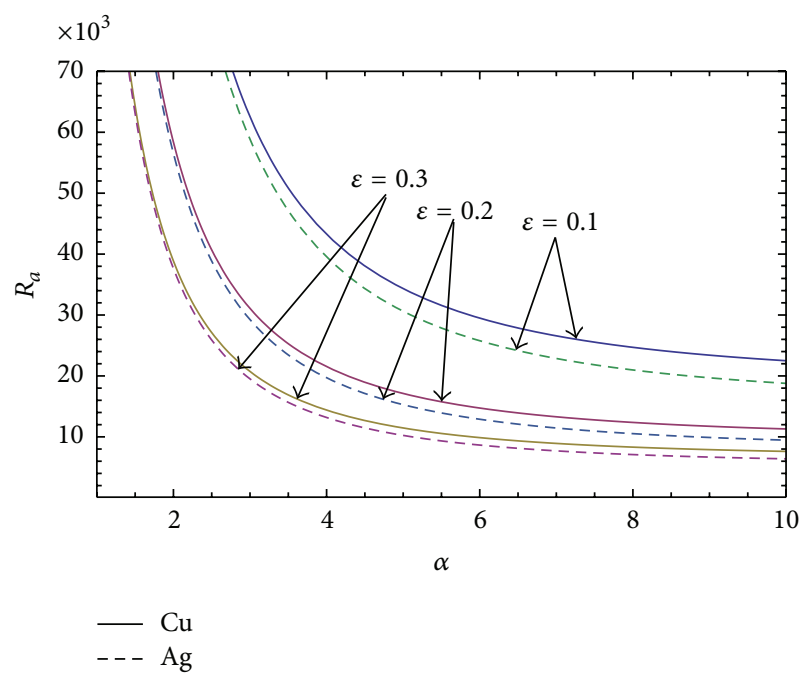

FIgURE 3: Effect of $\varepsilon$ on metallic nanoparticles.

diffusivity ratio for typical nanofluid is fixed. Further, metallic and nonmetallic (semiconducting) nanofluids have been considered with the existing data available in the literature to investigate the stability problem. We fix other parameters as Le $=2, \varepsilon=0.1, \mathrm{Rs}=200, S_{C T}=1, S_{T C}=0.001$, and $Q=400$.

Figures 1 and 2 illustrate the impact of Chandrasekhar number on the Rayleigh number for metals $(\mathrm{Cu}, \mathrm{Ag})$ and nonmetals $\left(\mathrm{TiO}_{2}, \mathrm{SiO}_{2}\right)$ in water based nanofluids. The value of $R_{a}$ increases appreciably with the increase in $Q$. Thus magnetic field delays the onset of convection in a binary nanofluid layer system and this stabilizing effect is more pronounced for the case of nonmetallic nanofluids.

Figures 3 and 4 show the effect of porosity on the system. The value of $R_{a}$ decreases with the increase in $\varepsilon$ and hence porosity hastens the onset of instability. The Rayleigh number for nonmetallic nanofluids is higher than that for metallic nanofluids. To assess the influence of solute concentration difference on the stability of the fluid layer system, it is necessary to note that the solute concentration difference is entering in the expression for Rayleigh number through solute parameters ( $\left.\mathrm{Rs}, S_{C T}, S_{T C}\right)$. With an increase in $\Delta C\left(C_{1}-C_{0}\right), R_{a}$ increases as shown in Figures 5 and 6 . Thus solute concentration difference on the boundaries stabilizes the binary nanofluid layer system.

Further, the destabilizing impact of nanoparticle volume fraction difference $\Delta \phi\left(\phi_{1}-\phi_{0}\right)$ (appears through $\mathrm{Rn}$ and $N_{A}$ ) on the fluid layer is depicted in Figures 7 and 8. This destabilizing influence is more pronounced for metallic nanofluids due to their higher densities as compared to semiconducting nanoparticles. 
TABLE 2: Nanofluidparameters for metallic/nonmetallic nanoparticles in water based nanofluids under consideration.

\begin{tabular}{lcccc}
\hline Nanofluid parameters & Cu-water nanofluid & Ag-water nanofluid & $\mathrm{SiO}_{2}$-water nanofluid & TiO -water nanofluid $^{-}$ \\
\hline $\mathrm{Rn}$ (nanoparticle Rayleigh number) & 0.392 & 0.465 & 0.0785 & 0.159 \\
$N_{A}$ (diffusivity ratio) & 0.5 & 0.5 & 17.5 & 20 \\
\hline
\end{tabular}

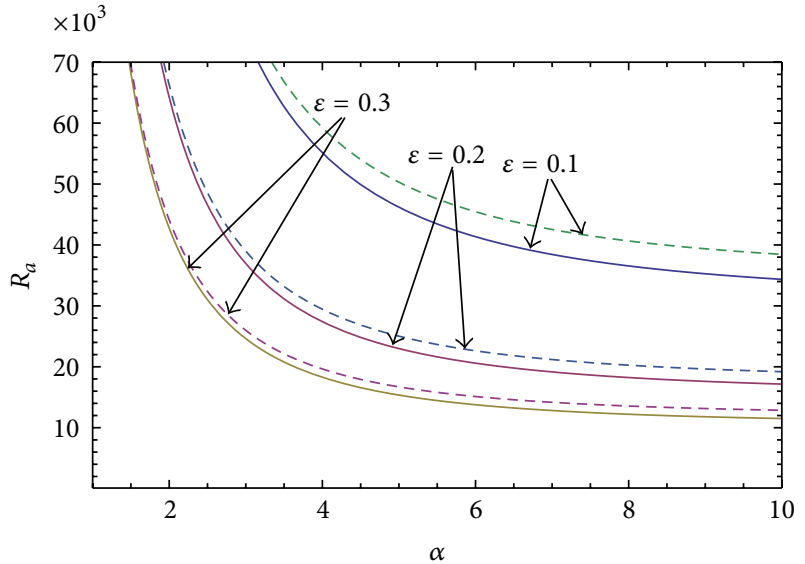

$-\mathrm{TiO}_{2}$

-.- $\mathrm{SiO}_{2}$

FIGURE 4: Effect of $\varepsilon$ on semiconducting nanoparticles.

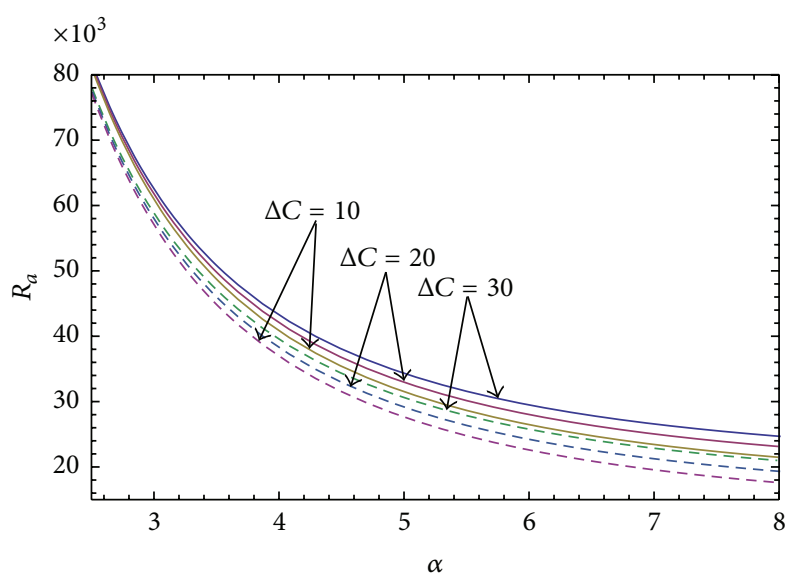

$\begin{aligned} &- \mathrm{Cu} \\ &---\mathrm{Ag}\end{aligned}$

FIgURE 5: Effect of $\Delta C$ on metallic nanoparticles.

Let us study the influence of Dufour and Soret parameters arising due to the presence of solute in the fluid layer which otherwise were assumed to be negligible in Section 4 to get analytical results. Dufour parameter stabilizes the system for both metals and nonmetals almost at the same rate (Figures 9 and 10) while destabilizing influence of Soret parameter on the stability is so small that it is not depicted in Figures 11 and 12. Interestingly, the stabilizing influence of nonmetals in binary nanofluids is more than that of metals. This may be due to the fact that density of nonmetals is much lower than metals. Also, It is noteworthy that copper-water nanofluid is

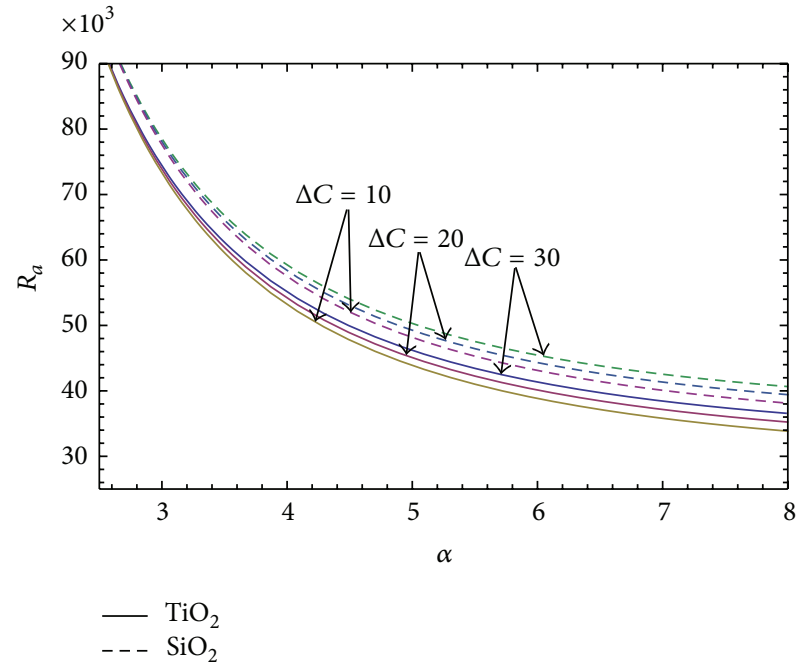

FigurE 6: Effect of $\Delta C$ on semiconducting nanoparticles.

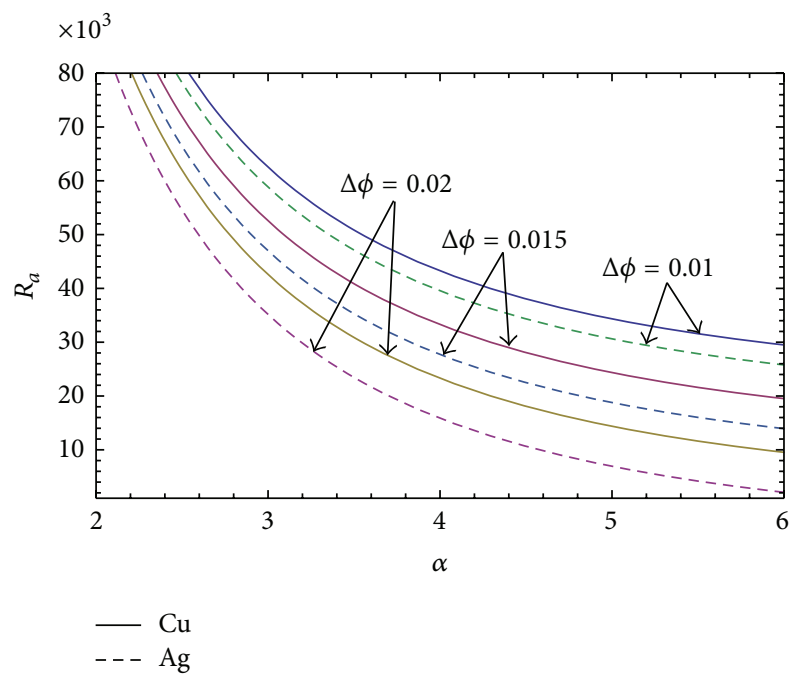

Figure 7: Effect of $\Delta \phi$ on metallic nanoparticles.

more stable than silver-water nanofluid (refer to Figures 1, 3, $5,7,9$, and 11) and silicon oxide-water nanofluid is more stable than titanium oxide-water nanofluid (refer to Figures 2, 4, 6, 8,10 , and 12).

\section{Conclusions}

The influence of magnetic field on the stability of binary nanofluid layer in porous medium is investigated using normal mode technique and Galerkin method. It is assumed that the temperature, nanoparticle volume fraction, and 


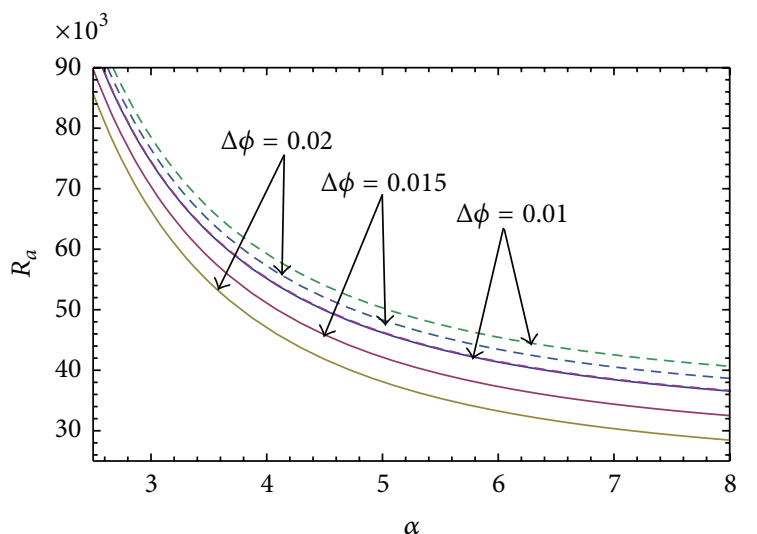

$-\mathrm{TiO}_{2}$

- - $\mathrm{SiO}_{2}$

FIGURE 8: Effect of $\Delta \phi$ on semiconducting nanoparticles.

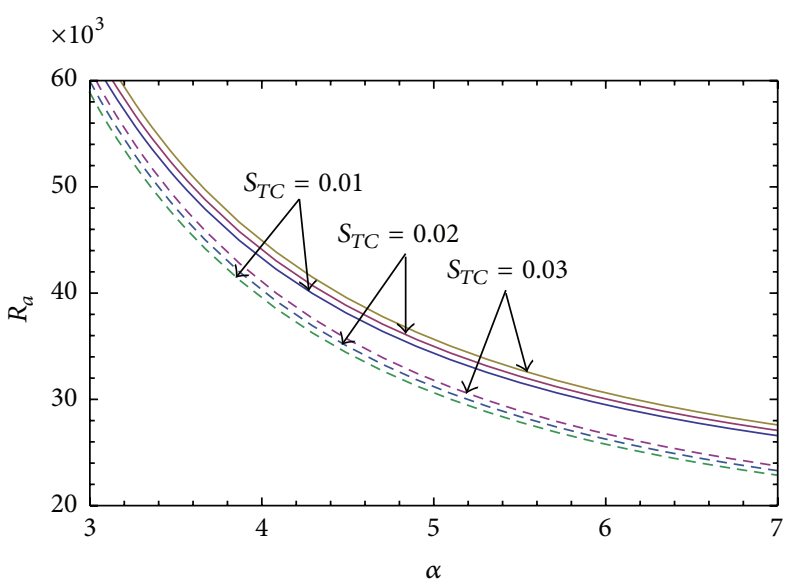

$-\mathrm{Cu}$

-.- Ag

FIgURE 9: Effect of $S_{T C}$ on metallic nanoparticles.

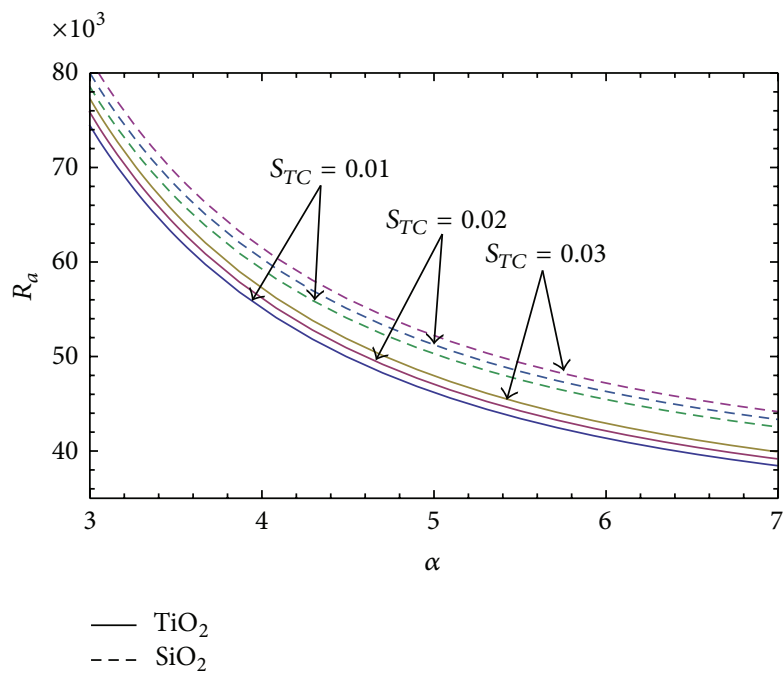

Figure 10: Effect of $S_{T C}$ on semiconducting nanoparticles.

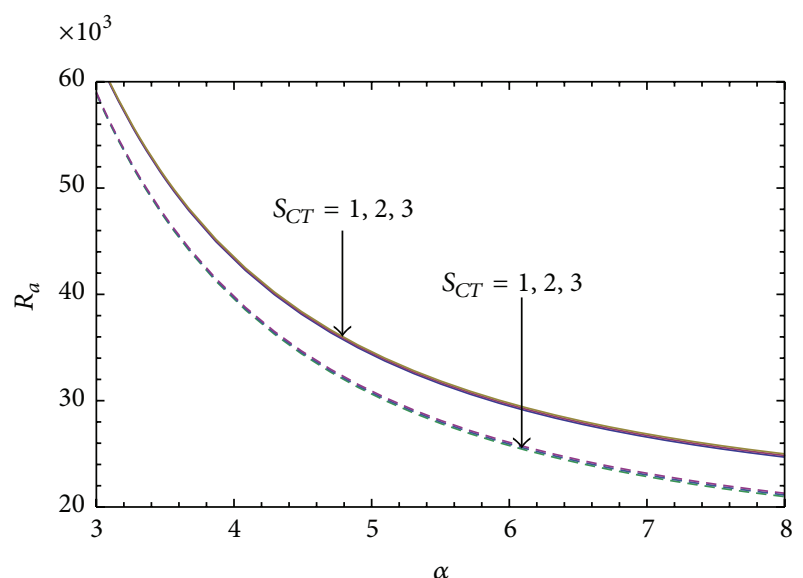

$$
\begin{array}{cc}
- \\
--\mathrm{Ag}
\end{array}
$$

FIGURE 11: Effect of $S_{C T}$ on metallic nanoparticles.

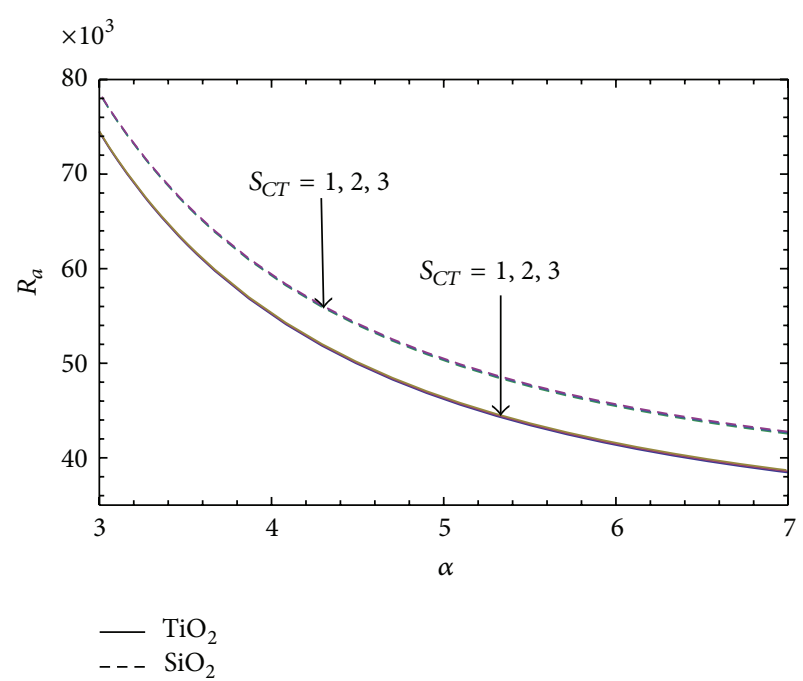

FIGURE 12: Effect of $S_{C T}$ on semiconducting nanoparticles.

solute concentration are constant along the walls. It is found that the regular cross-diffusion terms dominate the nanofluid terms. Out of the two nanoscale effects, thermophoresis is more responsible to initiate instability as compared to Brownian motion. The critical wave number rises with the rise in Chandrasekhar number and decreases with the rise in porosity while it is independent of the presence of solute and nanoparticles in the fluid layer. Influence of density of nanoparticles on the stability is more than their conductivity. The stability of the system increases/decreases with the increase in size of nanoparticles for bottom/top heavy distribution of nanoparticles in the fluid layer. The presence of nanoparticles on the top of the layer makes it less stable and oscillatory motions are not possible. Also, distribution of nanoparticles at the top of the layer makes system so unstable that the applied magnetic field must be increased or temperature at the bottom of the layer must be decreased relative to the top for neutral stability. Nonmetals stabilize the water 
based fluid layer more than metals. Copper-nanofluid is more stable than silver-water while silicon oxide-water nanofluid is more stable than titanium oxide-water nanofluid. Porosity destabilizes the system and solute concentration difference on the boundaries stabilizes it slightly. The destabilizing effect of nanoparticle volume fraction difference is more for metals than semiconductors due to their higher densities. Dufour parameter stabilizes the system while destabilizing influence of Soret parameter is almost negligible. Magnetic field is found to have a strong stabilizing impact on the fluid layer.

\section{Competing Interests}

The authors declare that they have no competing interests.

\section{References}

[1] H. Bénard, "Les Tourbillions Cellulaires dans une Nappe Liquide," Revue Générale des Sciences, vol. 11, pp. 1261-1271, 1900.

[2] L. Rayleigh, "On convection currents in a horizontal layer of fluid, when the higher temperature is on the underside," Philosophical Magazine, vol. 6, no. 32, pp. 529-546, 1916.

[3] S. Chandrasekhar, Hydrodynamic and Hydromagnetic Stability, Dover Publications, New York, NY, USA, 1981.

[4] M. E. Stern, “The 'salt-fountain' and thermohaline convection," Tellus, vol. 12, no. 2, pp. 172-175, 1960.

[5] S. Choi, "Enhancing thermal conductivity of fluids with nanoparticles," in Development and Applications of NonNewtonian Flows, D. A. Siginer and H. P. Wang, Eds., vol. 66, ASME FED-231/MD, pp. 99-105, ASME, 1995.

[6] H. Masuda, A. Ebata, K. Teramae, and N. Hishinuma, "Alteration of thermal conductivity and viscosity of liquid by dispersing ultra-fine particles. dispersion of $\mathrm{Al}_{2} \mathrm{O}_{3}, \mathrm{SiO}_{2}$ and $\mathrm{TiO}_{2}$ ultra-fine particles," Netsu Bussei, vol. 7, no. 4, pp. 227-233, 1993.

[7] J. A. Eastman, S. U. S. Choi, S. Li, W. Yu, and L. J. Thompson, "Anomalously increased effective thermal conductivities of ethylene glycol-based nanofluids containing copper nanoparticles," Applied Physics Letters, vol. 78, no. 6, pp. 718-720, 2001.

[8] P. Keblinski, S. R. Phillpot, S. U. S. Choi, and J. A. Eastman, "Mechanisms of heat flow in suspensions of nano-sized particles (nanofluids)," International Journal of Heat and Mass Transfer, vol. 45 , no. 4 , pp. 855-863, 2001.

[9] W. Wang, L. Lin, Z. X. Feng, and S. Y. Wang, "A comprehensive model for the enhanced thermal conductivity of nanofluids," Journal of Advanced Research in Physics, vol. 3, no. 2, Article ID 021209, 2012.

[10] J. Buongiorno, "Convective transport in nanofluids," ASME Journal of Heat Transfer, vol. 128, no. 3, pp. 240-250, 2006.

[11] D. Y. Tzou, "Thermal instability of nanofluids in natural convection," International Journal of Heat and Mass Transfer, vol. 51, no. 11-12, pp. 2967-2979, 2008.

[12] D. A. Nield and A. V. Kuznetsov, "Thermal instability in a porous medium layer saturated by a nanofluid," International Journal of Heat and Mass Transfer, vol. 52, no. 25-26, pp. 57965801, 2009.

[13] A. V. Kuznetsov and D. A. Nield, "Thermal instability in a porous medium layer saturated by a nanofluid: Brinkman model," Transport in Porous Media, vol. 81, no. 3, pp. 409-422, 2010.
[14] U. Gupta, J. Ahuja, and R. K. Wanchoo, "Magneto convection in a nanofluid layer," International Journal of Heat and Mass Transfer, vol. 64, pp. 1163-1171, 2013.

[15] N. Rudraiah, R. M. Barron, M. Venkatachalappa, and C. K. Subbaraya, "Effect of a magnetic field on free convection in a rectangular enclosure," International Journal of Engineering Science, vol. 33, no. 8, pp. 1075-1084, 1995.

[16] T. Hayat, R. Sajjad, Z. Abbas, M. Sajid, and A. A. Hendi, "Radiation effects on MHD flow of Maxwell fluid in a channel with porous medium," International Journal of Heat and Mass Transfer, vol. 54, no. 4, pp. 854-862, 2011.

[17] M. Sheikholeslami, M. M. Rashidi, T. Hayat, and D. D. Ganji, "Free convection of magnetic nanofluid considering MFD viscosity effect," Journal of Molecular Liquids, vol. 218, pp. 393399, 2016.

[18] M. Sheikholeslami, T. Hayat, and A. Alsaedi, "MHD free convection of Al2O3-water nanofluid considering thermal radiation: a numerical study," International Journal of Heat and Mass Transfer, vol. 96, pp. 513-524, 2016.

[19] R. Chand and G. C. Rana, "On the onset of thermal convection in rotating nanofluid layer saturating a Darcy-Brinkman porous medium," International Journal of Heat and Mass Transfer, vol. 55, no. 21-22, pp. 5417-5424, 2012.

[20] B. S. Bhadauria and S. Agarwal, "Natural convection in a nanofluid saturated rotating porous layer: a nonlinear study," Transport in Porous Media, vol. 87, no. 2, pp. 585-602, 2011.

[21] A. V. Kuznetsov and D. A. Nield, "The onset of double-diffusive nanofluid convection in a layer of a saturated porous medium," Transport in Porous Media, vol. 85, no. 3, pp. 941-951, 2010.

[22] U. Gupta, J. Sharma, and R. K. Wanchoo, “Thermosolutal convection in a horizontal nanofluid layer: introduction of oscillatory motions," in Proceedings of the Recent Advances in Engineering and Computational Sciences (RAECS '14), pp. 1-6, IEEE, Chandigarh, India, March 2014.

[23] D. Yadav, G. S. Agrawal, and R. Bhargava, "The onset of convection in a binary nanofluid saturated porous layer," International Journal of Theoretical and Applied Multiscale Mechanics, vol. 2, no. 3, pp. 198-224, 2012.

[24] U. Gupta, J. Sharma, and V. Sharma, "Instability of binary nanofluids with magnetic field," Applied Mathematics and Mechanics, vol. 36, no. 6, pp. 693-706, 2015.

[25] M. Turkyilmazoglu, "Exact analytical solutions for heat and mass transfer of MHD slip flow in nanofluids," Chemical Engineering Science, vol. 84, pp. 182-187, 2012. 


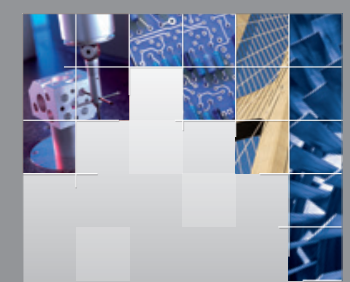

\section{Enfincering}
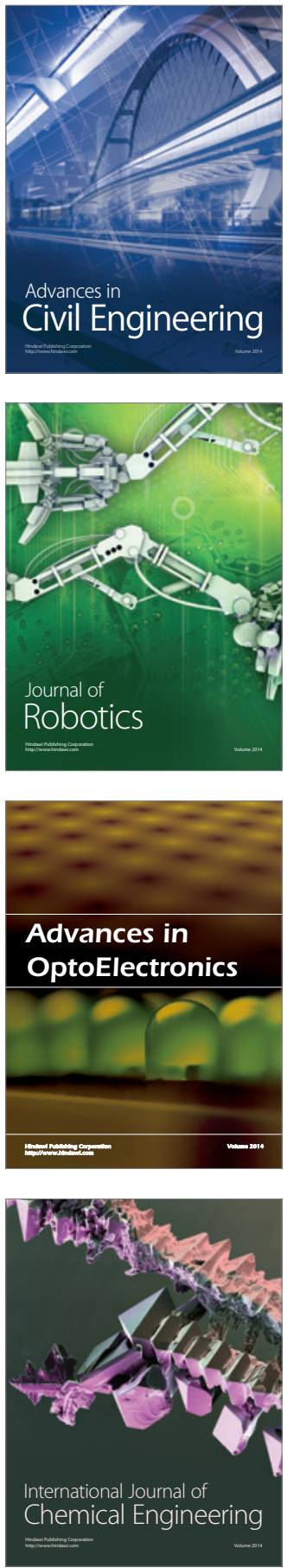

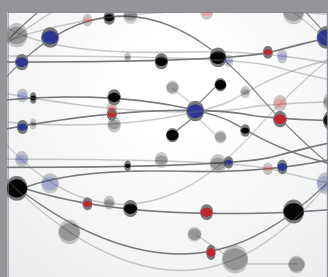

The Scientific World Journal

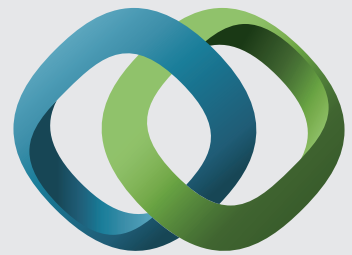

\section{Hindawi}

Submit your manuscripts at

http://www.hindawi.com
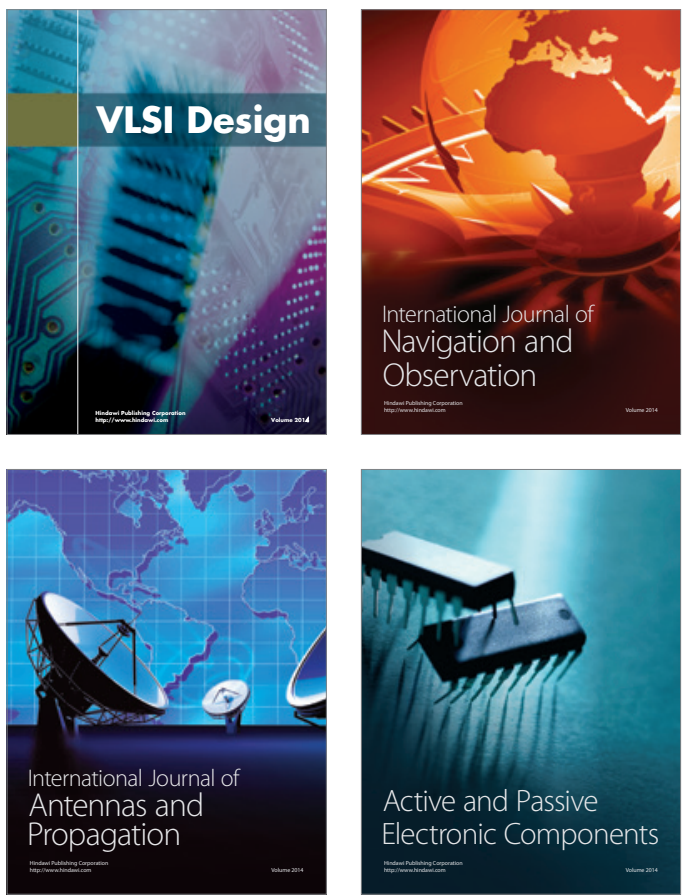
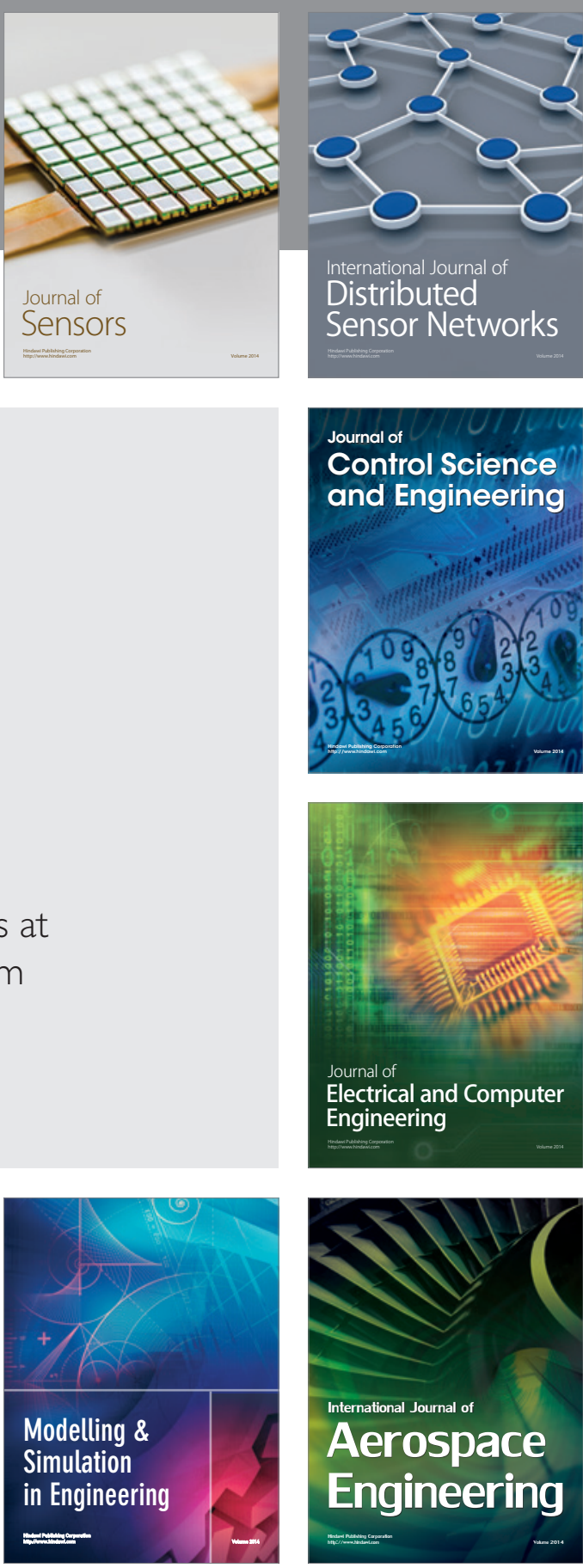

International Journal of

Distributed

Sensor Networks

Journal of

Control Science

and Engineering
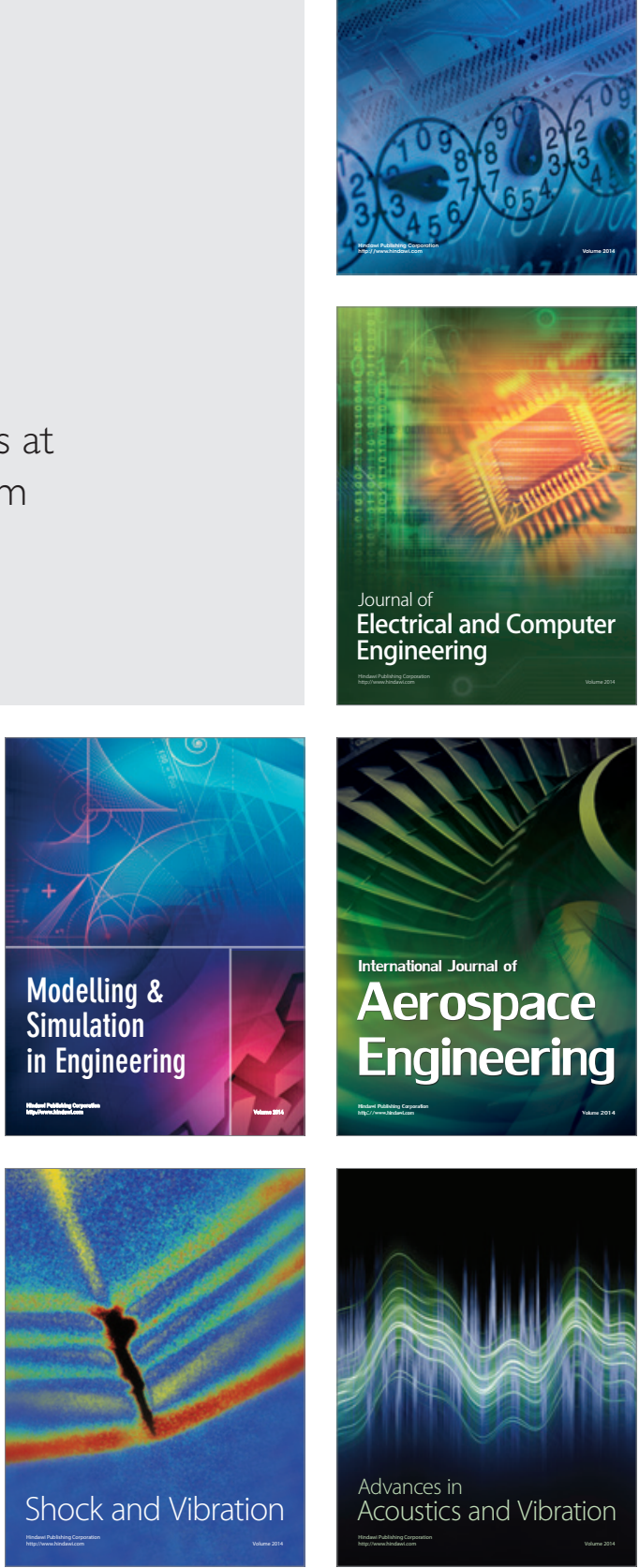